Nervenarzt 2020 • 91:872-874

https://doi.org/10.1007/s00115-020-00992-z

(c) Springer Medizin Verlag GmbH, ein Teil von Springer Nature 2020

\section{Redaktion}

M. Peeters | I. Lork, Berlin

A. Heinz, Berlin (V.i.S. d.P.)

DGPPN e.V. Geschäftsstelle

Reinhardtstr. 27 B

10117 Berlin
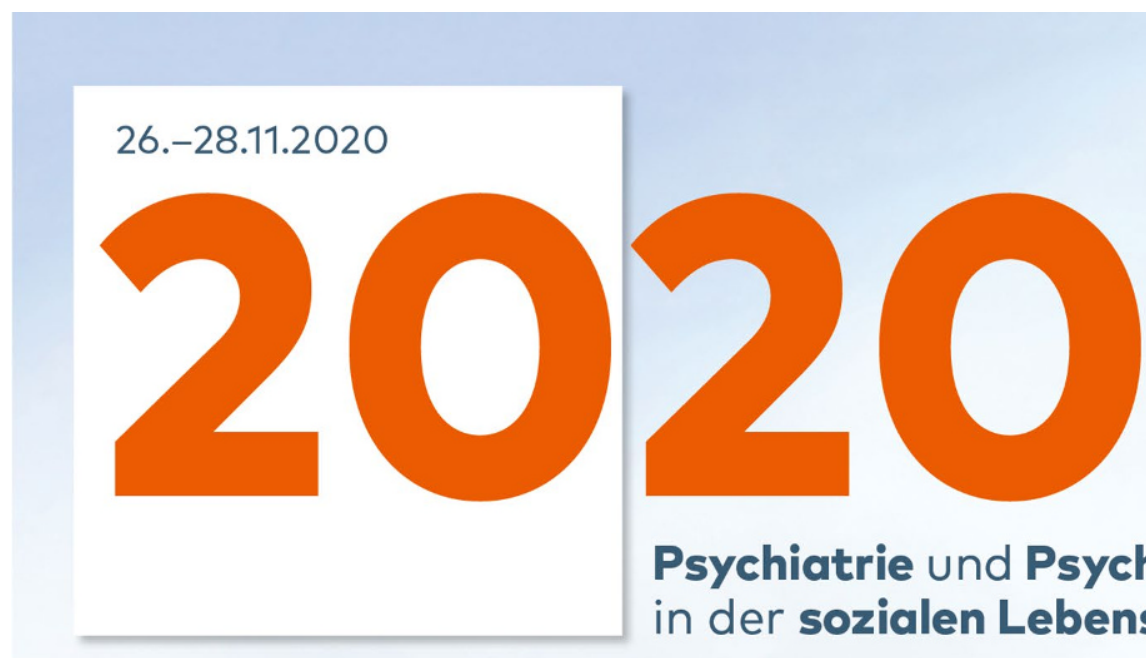

\title{
Psychiatrie und Psychotherapie
} in der sozialen Lebenswelt

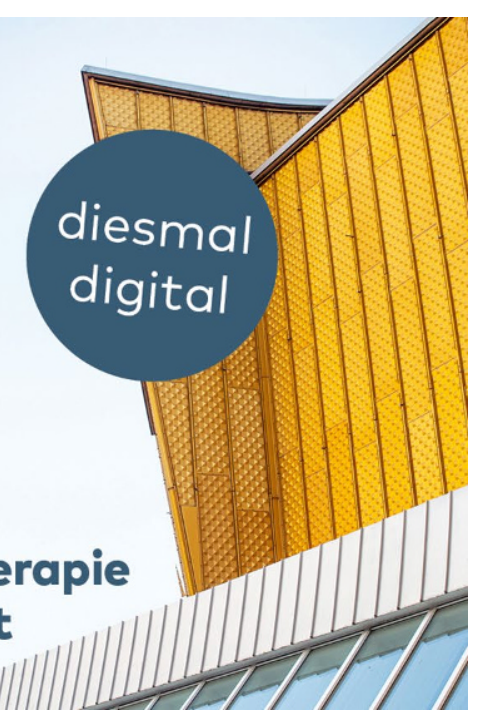

\section{DGPPN Kongress 2020: Top-Speaker mit dabei}

Der DGPPN Kongress in digitaler Form bietet vom 26. bis 28. November 2020 für angemeldete Teilnehmer auf dem Kongressportal ein wissenschaftliches Programm der Extraklasse mit über 200 Sessions. Die DGPPN bedankt sich für die vielen Einreichungen, die den Kongress auch in diesem Jahr unter den besonderen Umständen zu einem Spitzentreffen der psychischen Gesundheit machen werden. Angemeldeten Teilnehmern stehen nach erfolgreichem Log-in auf der Kongresswebsite verschiedene Kanäle zur Auswahl, in denen die Symposien, Lectures, Diskussionsforen, State-of-the-Art- und Präsidentensymposien ausgestrahlt werden - ähnlich wie beim TV-Programm.

Der detaillierte Programmplan mit allen Sendezeiten ist ab
Ende September online einsehbar. Die Kongressgebühren betragen in allen Kategorien $50 \%$ der Frühbucherrate. Die gesamte Gebührenliste sowie alle Informationen zur Registrierung sind auf www.dgppnkongress.de veröffentlicht. Die Anmeldung zum Kongress ist sowohl jetzt als auch während der Kongresstage kurzfristig möglich.

\section{Renommierte Persönlich- keiten}

Im Mittelpunkt des wissenschaftlichen Programms stehen wie immer brandaktuelle Themen. Das Motto „Psychiatrie und Psychotherapie in der sozialen Lebenswelt" umfasst die unterschiedlichsten Aspekte. Neben Martin Paulus, Amma Yeboah und Gerd Kempermann haben sich weitere Top-Speaker angekündigt.
Daniel Weinberger

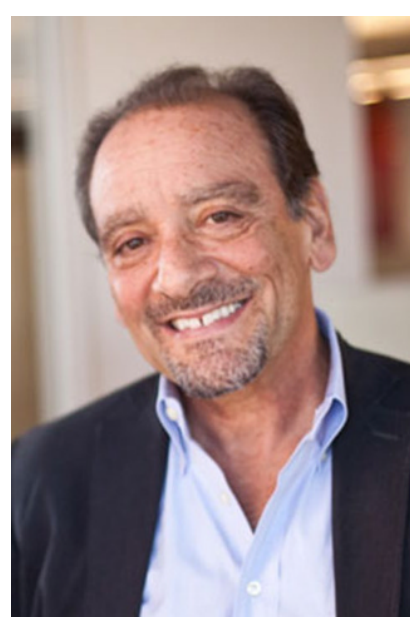

Der führende Neuro-Wissenschaftler ist bekannt für seine Arbeit zur Identifizierung genetischer Faktoren und biochemischer Mechanismen bei der Entstehung psychischer Erkrankungen, insbesondere der Schizophrenie. In Zukunft könnten Weinbergers Erkenntnisse dabei helfen, das individuelle Risiko für psychische Erkrankungen besser abzuschätzen und womöglich schon im Mutterleib $\mathrm{zu}$ minimieren, was einem wissenschaftlichen Durchbruch gleichkommt.

\section{Auf einen Blick}

- 26. bis 28. November 2020

- Zugang zum digitalen Kongress über www.dgppnkongress.de

- vorherige Registrierung erforderlich

- verschiedene Kanäle anwählbar

- Live-Interaktion mit den Vortragenden

- Kongress + Video-on-Demand-Angebot = über 80 CME-Punkte möglich

- Spezialrate für alle: $50 \%$ der Frühbucherrate 


\section{Eva Illouz}

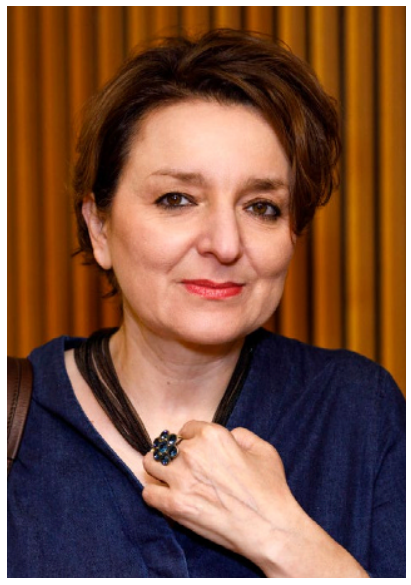

Die international bekannte Professorin für Soziologie und Anthropologie zählt zu den bedeutendsten Intellektuellen unserer Zeit. Illouz lehrt unter anderem an der Hebräischen Universität in Jerusalem. $\mathrm{Zu}$ ihren Forschungsschwerpunkten zählen die Soziologie der Emotionen, der Konsumgesellschaft und der Medienkultur. In ihren viel diskutierten Publikationen untersucht Eva Illouz die Liebe in Zeiten des Kapitalismus. In ihrer digitalen Lecture spricht sie über Emotionen im Zusammenhang mit Rassismus.

\section{Martin Fischer}

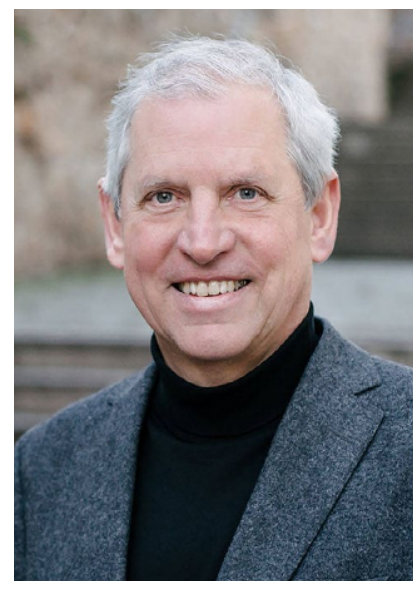

Das Konzept der „Rasse“ ist das Ergebnis von Rassismus und nicht dessen Voraussetzung. Dass es keinerlei biologische Begründung für den Begriff „Menschenrasse" gibt, hat der Evolutionsforscher und Zoologe Martin Fischer zuletzt in der ,Jenaer Erklärung " erläutert. Dort heißt es: „Der Nichtgebrauch des Begriffes Rasse sollte heute und zukünftig zur wissenschaftlichen Redlichkeit gehören."

\section{Orientierungshilfen in post- akuten Pandemie-Zeiten}

Durch die SARS-CoV-2-Pandemie waren tiefgreifende Umstrukturierungen in der gesamten medizinischen Versorgung notwendig. Auch psychiatrische Kliniken wurden verpflichtet, Kapazitäten freizuhalten und entsprechende Hygienevorschriften zu befolgen und dabei gleichzeitig ihren Versorgungsauftrag zu erfüllen. Psychiatrische Praxen und psychiatrische Institutsambulanzen arbeiten ihrerseits aktuell unter verschärften Bedingungen, insbesondere in Hinblick auf Hygienemaßnahmen und das erweiterte Angebot im Rahmen der digitalen und mobilen Arzt-Patienten-Kommunikation. Für beide Bereiche ergaben sich Herausforderungen, die nach wie vor bestehen. Auch mittelfristig muss im Fachgebiet der Psychiatrie und Psychotherapie zwischen erweiterten Versorgungsangeboten und dem Schutz von Mitarbeitern und Patienten sorgsam abgewogen werden. Der DGPPN-Vorstand hat deshalb Orientierungshilfen für die ambulante und stationäre Versorgung psychisch Kranker in der postakuten Phase der SARSCoV-2-Pandemie erarbeitet.

Die vollständige Stellungnahme auf dgppn.de

\section{Nein zu Rassismus}

Menschen anderer Hautfarbe erfahren immer noch überall auf der Welt, auch in Deutschland, Rassismus, Diskriminierung und Ausgrenzung. Rassistische Diskriminierung und Ausgrenzung tragen auch zu psychischen Erkrankungen bei. Psychisch krank zu sein kann wiederum immer noch zu gesellschaftlicher Ausschließung führen. Ein Teufelskreis und eine unheilvolle Situation für alle Betroffenen. Aus aktuellem Anlass hat der DGPPN-Vorstand zusammen mit dem Fachreferat „Interkulturelle Psychiatrie und
Psychotherapie, Migration" und einer Arbeitsgruppe von Experten mit Bezug zu den am stärksten betroffenen Communities eine Erklärung herausgebracht. Damit werden Politik, Institutionen und Wissenschaftsförderung aufgerufen, das Thema der sozialen Ausschließung, alle Formen der Diskriminierung und des Rassismus und deren Bedeutung für die psychische Gesundheit der Betroffenen verstärkt auf die Agenda zu setzen.

Die aktuelle Erklärung jetzt auf dgppn.de

\section{Digitales Hauptstadt- symposium: das Prinzip Trialog}

Die Versorgung psychisch erkrankter Menschen in Deutschland wurde mit der Psychiatrie-Enquête ab Mitte der 1970er Jahre ganz grundlegend reformiert. Menschen mit psychischen Erkrankungen sollten aus der Verwahrpsychiatrie in die Gesellschaft zurückgeholt werden, am Leben teilhaben, ihre Autonomie geachtet und ihre Versorgung wohnortnah, in der Gemeinde verankert sein. Heute, vierzig Jahre später und unter ganz neuen gesellschaftlichen Voraussetzungen und Wertevorstellungen stellt sich die Frage: Wie geht es mit der Psychiatriereform weiter? Klar ist schon jetzt, dass eine moderne Psychiatrie trialogisch - also von Betroffenen, Angehörigen und Profis - getragen werden muss. Das kommende digitale Hauptstadt- symposium der DGPPN macht deshalb den Trialog zum Grundprinzip: Aus trialogischer Sicht werden die aktuelle Situation von Psychiatrie und Psychotherapie im Kontext sozialer Lebenswelten, Menschenrechte und Versorgung beleuchtet. Die Referenten zeigen auf, wie eine zukunftsfähige psychiatrische Versorgung aussehen muss. Auch das zweite DGPPN-Hauptstadtsymposium in diesem Jahr wird als OnlineSeminar stattfinden. Eine Zertifizierung ist bei der zuständigen Ärztekammer beantragt. Save the date!

Wann: 27. bis 29. Oktober 2020 jeweils 17.00-18.00 Uhr Die Teilnahme ist kostenfrei. Informationen und Anmeldung über dgppn.de 


\section{Thema: Suizidassistenz}

Mit seinem Urteil vom 26. Februar 2020 hat das Bundesverfassungsgericht (BVerfG) das bisherige Verbot geschäftsmäBiger Suizidhilfe des $\$ 217$ StGB für nichtig erklärt. Zugleich benannte es detaillierte Rahmenbedingungen für eine gesetzliche Neuregelung, um den Gefahren einer unregulierten Suizidhilfe $\mathrm{zu}$ begegnen. Insbesondere an die Freiverantwortlichkeit, also die Selbstbestimmtheit, Dauerhaftigkeit und Ernsthaftigkeit des Suizidwunsches (BVerfG Rn. 241-247), sind nach den Vorgaben des BVerfG hohe Anforderungen zu stellen. Die DGPPN betont in diesem Kontext, dass
Suizidassistenz keine ärztliche Aufgabe ist. Ein Mittel zum Suizid zu beschaffen und bereitzustellen, stellt keine medizinische Behandlungsoption dar. Deshalb sollen Ärzte keine Beihilfe zum assistierten Suizid leisten. Aus Sicht der DGPPN sollte ein "legislatives Schutzkonzept" in dem vom Gericht festgelegten Rahmen die Selbstbestimmung der Betroffenen respektieren, sie aber vor einem irreversiblen Schritt wie dem Suizid schützen, wenn ihre Selbstbestimmung erheblich eingeschränkt ist.

Die vollständige Pressemitteilung unter dgppn.de $\rightarrow$ Presse $\rightarrow$ Pressemitteilungen 